\title{
Negativity for Specific Autoantibodies in Patients with Type 1 Diabetes That Developed on a Background of Common Variable Immunodeficiency
}

\author{
Tomáš Milota $^{a}$ Zdeněk Šumník $^{\mathrm{b}}$ Barbora Obermannováb Pavlína Králíčkovác Karel Vondrák ${ }^{b}$

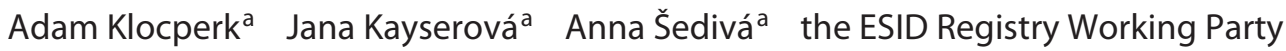 \\ Departments of ${ }^{\mathrm{a}}$ Immunology and ${ }^{\mathrm{b}}$ Paediatrics, 2nd Faculty of Medicine, Charles University, University Hospital \\ Motol, Prague, and ' Department of Clinical Immunology and Allergology, Hradec Králové University Hospital, \\ Hradec Králové, Czech Republic
}

\section{Established Facts}

- Type 1 diabetes (T1D) has only been described as an unusual autoimmune complication of common variable immunodeficiency (CVID) in several case reports.

- T1D can develop in an environment of severe antibody deficiency, in the absence of specific T1D-associated antibodies.

\section{Novel Insights}

- Eleven previously published case reports that described an association between T1D and CVID are reviewed.

- New epidemiological data gained from the European Society for Immunodeficiencies Registry suggest that T1D occurs in $1.5 \%$ of CVID patients $(1,500 / 100,000)$.

- Possible mechanisms of T1D development are discussed from the perspective of underlying humoral immunodeficiency.

- Based on our data, an important effect of immunoglobulin substitution therapy on the course of both CVID and T1D is assumed and discussed.

\section{Key Words}

Type 1 diabetes · Common variable immunodeficiency · Autoimmunity

\section{Abstract \\ Common variable immunodeficiency (CVID) is a heteroge- neous group of disorders characterized by disturbed anti-}

body production and a dysregulated immune system. Aside from recurrent infections, the most common complications of CVID are autoimmune complications, particularly autoimmune cytopenias. To date, type 1 diabetes mellitus (T1D) in combination with CVID has only been described as an unusual complication in several reports, but the true incidence of T1D with CVID remains unknown. We describe 2 patients with a combination of T1D and CVID with serious impair-

\section{KARGER 125}

(c) 2016 S. Karger AG, Basel

$1018-2438 / 16 / 1683-0197 \$ 39.50 / 0$

E-Mail karger@karger.com

www.karger.com/iaa
Correspondence to: Dr. Tomáš Milota

Department of Immunology, 2nd Faculty of Medicine

Charles University, University Hospital Motol

V Úvalu 84, CZ-150 06 Prague (Czech Republic)

E-Mail tomas.milota@fnmotol.cz 
ment of antibody production. We also provide a review of the available literature. T1D-specific insulin autoantibodies and autoantibodies to glutamic acid decarboxylase and tyrosine phosphatase IA2 were not detected in either of our patients at the time of diagnosis or during the course of the disease. In both cases, T1D manifestation and diagnosis preceded the discovery of CVID by several years. Following the diagnosis of immunodeficiency and the start of immunoglobulin substitution therapy, their clinical status improved, manifesting as a lower frequency of infections and improved T1D control, with decreased glycosylated hemoglobin A1C values. Based on these reported cases, we assume that T1D might be more frequent than previously reported in patients with CVID. To verify the actual incidence of T1D among CVID patients, we searched the European Society for Immunodeficiencies Registry database, and found 25 cases of T1D in 1,671 listed CVID patients, suggesting a higher occurrence of T1D among CVID patients than previously thought. Early diagnosis and treatment of immunodeficiency improve both the prognosis and the course of CVID, reduce the frequency and severity of infections and may contribute to better management of T1D.

(c) 2016 S. Karger AG, Basel

\section{Introduction}

Type 1 diabetes mellitus (T1D) is an organ-specific autoimmune disease caused by an immune reaction that is directed against the beta cells of the pancreas. European and US epidemiological studies suggest the incidence of T1D to be approximately 15/100,000 (varying between $10 / 100,000$ and 53/100,000) with a prevalence of $182 / 100,000[1,2]$. The disease develops due to complex immune reactions that depend on both the genetic background of the patient and environmental influences; these reactions lead to disruption of the tolerance to pancreatic beta cell-specific antigens [3]. The autoimmune destruction of beta cells is a complex process involving $\mathrm{T}$ lymphocytes, particularly Th1 cells, although a Th17 response has recently been described as well [4]. It is clear, however, that other cell populations such as NK cells and dendritic cells and reactions of innate immunity are also involved in T1D development [5].

B lymphocytes also form an integral part of complex immune reactions that lead to T1D. The role of these cells as producers of specific antibodies that both precede and accompany the course of T1D [6] is undisputed [7]. Their necessity for the development of T1D has, however, been strongly challenged by a report of T1D devel- oping in a patient with a severe B lymphocyte immunodeficiency, or X-linked agammaglobulinemia (XLA), which blocks B lymphocyte development and maturation [8]. This case report and others have shown that T1D may develop on a background of severe humoral immunodeficiency, XLA or common variable immunodeficiency (CVID).

CVID is the name for a heterogeneous group of disorders that are characterized by impaired antibody production and immune system dysregulation. The incidence is estimated to be between $1 / 25,000$ and $1 / 50,000$ subjects [9]. CVID is caused by a heterogeneous and, as yet, imprecisely specified genetic background; the specific genetic causes have been elucidated in only a small fraction of CVID patients. These causes include mutations in the inducible costimulator (ICOS) molecule, which is expressed on $\mathrm{T}$ lymphocytes and participates in $\mathrm{T}$ - and B-lymphocyte interactions, mutations in the transmembrane activator and calcium modulator and cyclophilin ligand interactor (TACI) gene, the B lymphocyte membrane proteins CD19, CD20 and CD81 and a gene encoding the BAFF receptor or MutS homolog 5 (MSH5). Additionally, next-generation sequencing of samples from CVID patients recently revealed mutations in the genes encoding BTK, SH2D1A, CD40/CD40L, AID, CLEC16A and DOCK 2. Other possible genetic causes are under evaluation. Despite these efforts, we only know the exact etiology in approximately $10 \%$ of patients $[10,11]$. CVID is often accompanied by other complications which are usually autoimmune in nature. This autoimmunity typically manifests as hematologic disorders, such as autoimmune hemolytic anemia and thrombocytopenia, but an entire spectrum of other conditions (e.g. rheumatoid arthritis, autoimmune thyroiditis, primary biliary cirrhosis and systemic lupus erythematosus) has been reported as well [12]. Autoimmune complications are generally observed in $20-30 \%$ of CVID patients, but only a few cases of T1D in conjunction with CVID have been reported.

In this report, we describe 2 patients with a typical T1D clinical presentation but a complete absence of T1D-associated antibodies. Only later were these patients diagnosed with immunodeficiency that was classified as CVID, and serious impairment of or even an absence of specific antibody production. Additionally, we summarize previously published case reports [13-22] and a review [23], and report the results of a targeted search of the European Society for Immunodeficiencies (ESID) Registry database. 
Table 1. Laboratory findings

\begin{tabular}{lllc}
\hline & Patient 1 & Patient 2 & Reference value \\
\hline Immunologic parameters & & & $7.65-13.6 \mathrm{~g} / \mathrm{l}$ \\
IgG & 4.5 & 0.73 & $0.91-2.9 \mathrm{~g} / \mathrm{l}$ \\
IgA & 0.5 & 0.11 & $0.47-1.95 \mathrm{~g} / \mathrm{l}$ \\
IgM & 0.2 & 0.04 & $0-150 \mathrm{IU} / \mathrm{ml}$ \\
IgE & 1,530 & 25.3 & $>0.1 \mathrm{IU} / \mathrm{ml}$ \\
Tetanus-specific antibodies & 0.01 & 0.04 & $4.0-10.0$ \\
White blood cell count & 4 & 11.3 & $25-33 \%$ \\
Lymphocytes & 42.2 & 10.8 & $60-85 \%$ \\
CD3+ cells & 65 & 74 & $27-57 \%$ \\
CD4+ cells & 43 & 46 & $18-40 \%$ \\
CD8+ cells & 22 & 24 & $7-30 \%$ \\
CD19+ cells & 14 & 17 & $6-28 \%$ \\
NK cells & 13 & 5 & - \\
Screening panel of autoantibodies ${ }^{\mathrm{a}}$ & negative & negative & \\
T1D control parameters & & & $20-42 \mathrm{mmol} / \mathrm{mol}$ \\
Glyc. HbAlc & 100 & 120 & $2.8-8 \mathrm{mmol} / \mathrm{l}$ \\
\hline Renal function parameters & & & $19-62 \mu \mathrm{mol} / \mathrm{l}$ \\
Urea & 4.5 & 12.8 & $20-150 \mathrm{mg}$ \\
Creatinine & 79 & 742 & \\
Microalbuminuria & 6.4 & & \\
\hline
\end{tabular}

These laboratory parameters were established at the time of diagnosis of CVID and before the initiation of immunoglobulin replacement therapy (the corresponding reference values are also provided).

a Screening panel of autoantibodies includes ANA, ANCA, ASCA, IgG and IgA antiendomysial antibodies as well as anti-ds DNA, RF (IgG, IgA and IgM), anti-TPO, anti-TG and anti-ENA (Sm, SS-A, SS-B, Scl-70 and Jo-1) antibodies.

\section{Case Report}

\section{Case 1}

A 26-year-old male patient treated for T1D was referred to our department for evaluation of hypogammaglobulinemia, discovered during laboratory investigation of progressive cervical lymph node lymphadenopathy.

The patient's family history showed a significant number of autoimmune and allergic disorders. His father has been treated for multiple sclerosis, and his brother for atopic dermatitis and polyvalent allergy. The patient's prenatal and perinatal development had been free of complications. His first health issue appeared at 3 years of age, when he was diagnosed with intermittent allergic bronchial asthma and a polyvalent allergy.

At 6 years of age, the patient was admitted to the hospital for the treatment of hyperglycemia $(21.9 \mathrm{mmol} / \mathrm{l})$ with ketoacidosis, and he was diagnosed with T1D, based on laboratory examinations, with repeated negative findings for T1D-associated autoantibodies [insulin autoantibodies (IAA) and autoantibodies to glutamic acid decarboxylase (GAD) and tyrosine phosphatase IA2]. An intensified insulin regimen was started, with a total daily dose of $34 \mathrm{IU}(0.86 \mathrm{IU} / \mathrm{kg} /$ day). The glycosylated hemoglobin A1c (glyc. HbA1c) level before initiation of the regime was $108 \mathrm{mmol} / \mathrm{mol}$ (IFCC units) or 12\% (NGSP/DCCT units).
Since the age of 13 years, this patient had been repeatedly examined due to skin abscesses located on the auricle. Additionally, he had exhibited progressive cervical lymphadenopathy. Repeated histologic examinations of the lymphatic nodes upon extirpation revealed only unspecific reactive changes, and examination of specific antibodies also revealed nothing. A basic immunologic examination (table 1) was performed as part of a differential diagnosis and revealed hypogammaglobulinemia in the IgG, IgA and IgM classes. The patient was therefore referred to our department for a full immunologic examination, which verified the hypogammaglobulinemia (IgG $4.5 \mathrm{~g} / \mathrm{l}$, IgA $0.5 \mathrm{~g} / \mathrm{l}$ and IgM $0.2 \mathrm{~g} / \mathrm{l}$ ), significantly decreased levels of specific postvaccination antibodies (tetanus-specific antibodies $0.01 \mathrm{IU} / \mathrm{ml}$, mumps-specific antibodies $0.89 \mathrm{RU} / \mathrm{ml}$ and diphtheria-specific antibodies $0.07 \mathrm{IU} / \mathrm{ml}$ ) and increased $\mathrm{IgE}$ levels $(1,250 \mathrm{IU} / \mathrm{ml})$. The allergen-specific IgE results corresponded to a polyvalent allergy diagnosis (i.e. allergies to molds, tree and grass pollen, dust mites and cats). Spirometry did not show any pathologic ventilation parameters. Additionally, an autoantibody panel was negative (i.e. the expected result in a patient with humoral deficiency and impaired antibody production). An examination of cellular immunity, including B lymphocyte subpopulations (table 2), along with the hypogammaglobulinemia and impairment of specific-antibody production findings, led to a diagnosis of type II CVID via the Freiburg classification (fig. 1). Regular intravenous immunoglobulin substitution therapy was thus initiated. 


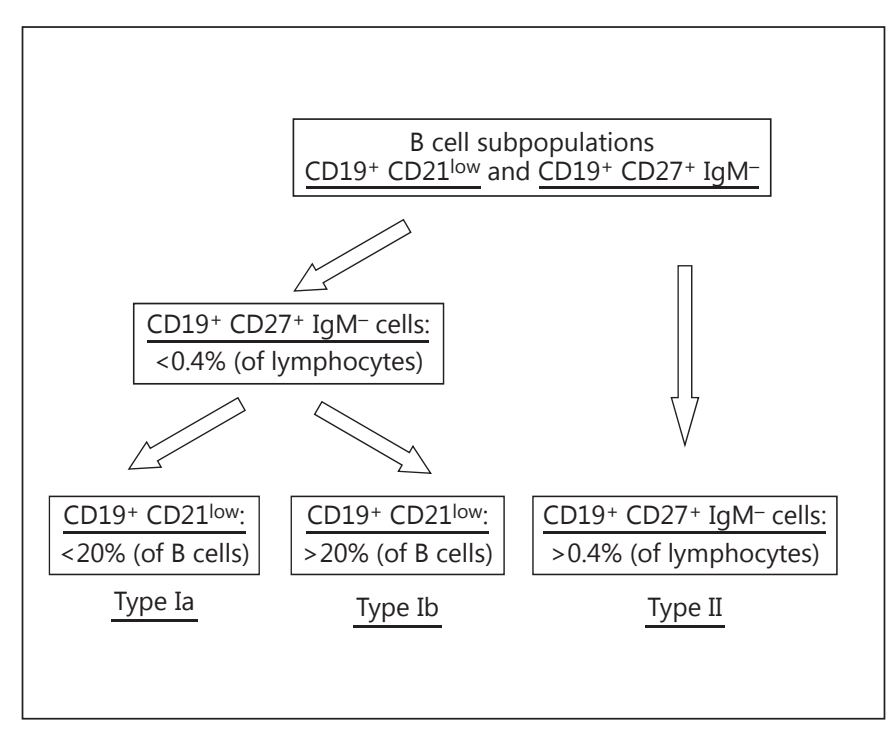

Fig. 1. Freiburg classification of CVID.

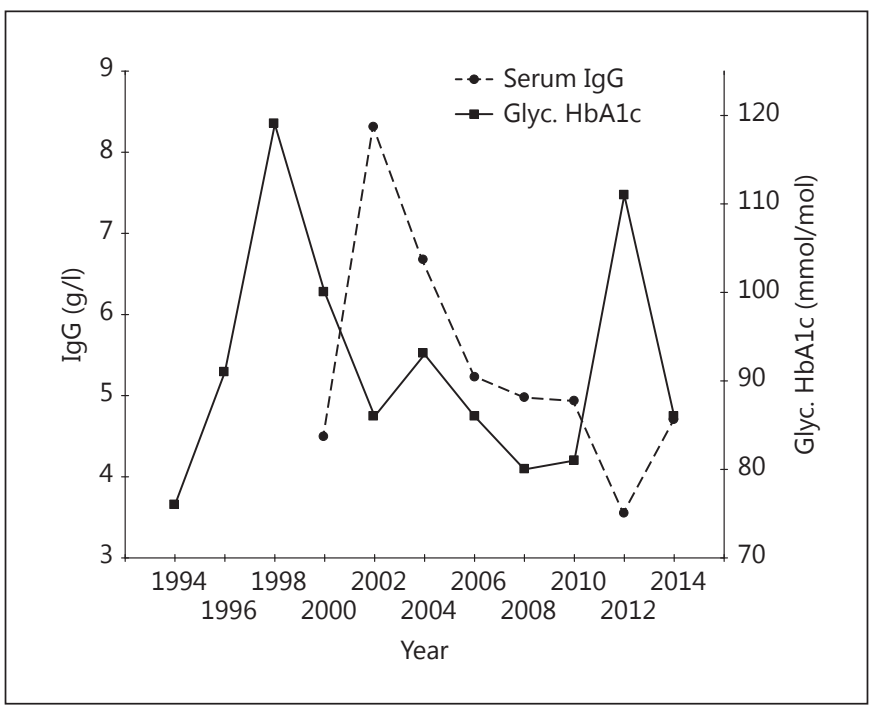

Fig. 2. Serum IgG and glyc. HbA1c levels in patient 1. Progression of glyc. HbA1c levels since T1D diagnosis in 1994 were compared with serum IgG levels after the CVID diagnosis in 2000 and the adjustment of immunoglobulin substitution to $10 \mathrm{~g}(164 \mathrm{mg} / \mathrm{kg})$ per month in 2012 .

Table 2. B cell subsets in our 2 patients

\begin{tabular}{|c|c|c|c|c|}
\hline B cell subset & Characteristics & Patient 1 & Patient 2 & Reference values $^{\mathrm{a}}$ \\
\hline $\mathrm{CD} 21^{\text {low }}, \mathrm{CD} 38^{\text {low }}$ & $\mathrm{CD} 19+, \mathrm{CD} 21^{\text {low }}, \mathrm{CD} 38^{\text {low }}$ & 7.34 & 2.31 & $4.4 \%(1.6-10)$ of B cells \\
\hline Plasmablasts & $\mathrm{CD} 19+, \mathrm{CD} 27+, \mathrm{CD} 38+$ & 0.922 & 1.18 & $1.1 \%(0.4-2.4)$ of B cells \\
\hline Marginal zone-like & $\mathrm{CD} 19+, \mathrm{CD} 27+, \mathrm{IgM}+, \mathrm{IgD}+$ & 14.5 & 13.7 & $11.1 \%(7.0-23.8)$ of B cells \\
\hline Transitional & $\mathrm{CD} 19+, \mathrm{CD} 27-, \mathrm{CD} 24+, \mathrm{CD} 38+$ & $7.26 \Delta$ & $17.1 \Delta$ & $2.5 \%(0.9-5.7)$ of B cells \\
\hline Naïve & $\mathrm{CD} 19+, \mathrm{CD} 27-, \mathrm{IgD}+$ & 60.9 & 70.9 & $65.5 \%(48.4-79.7)$ of B cells \\
\hline
\end{tabular}

This examination was performed in 2013. $\boldsymbol{\nabla}$ Decreased value; $\boldsymbol{\Delta}$ increased value.

a The median values, i.e. 5-95\% percentile range, are shown in parentheses.

Despite this regimen, we did not achieve satisfactory compensation for either the CVID (the IgG levels were repeatedly $<4 \mathrm{~g} / \mathrm{l}$, with an increased number of infections) or the T1D (glyc. HbAlc values were repeatedly $>100 \mathrm{mmol} / \mathrm{mol}$ or $>11.3 \%$ ). We therefore gradually increased the total immunoglobulin replacement therapy dose to $10 \mathrm{~g}(164 \mathrm{mg} / \mathrm{kg})$ per month and the insulin regime to a total daily dose of $71 \mathrm{IU}$ ( $1.16 \mathrm{IU} / \mathrm{kg} /$ day).

This adjustment led to an increase in the serum IgG level, a decreased number of infections and better compensation for the T1D (fig. 2). During the development of the disease, the patient also exhibited decreased numbers of B lymphocytes (fig. 3), along with a significant decrease in IgA and IgM levels. Additionally, the total IgE level normalized, and the specific IgE level decreased.

The current clinical state of our patient is satisfactory, with no long-term infections. There are no signs of progression of his cer- vical lymphadenopathy or any other complications. The compensation for T1D is satisfactory as well.

\section{Case 2}

An 18-year-old male patient who was being treated for T1D was referred to our department for evaluation of hypogammaglobulinemia, which was discovered during the investigation of a nosocomial lower respiratory tract infection that was acquired during his stay in the hospital.

This patient also presented with a significant family medical history. In particular, his grandparents on his father's side were being treated for type 2 diabetes with macrovascular complications and his older sister was being treated for bronchial asthma and autoimmune thyroiditis. The patient's perinatal and postnatal development had been normal. His first health issue appeared 


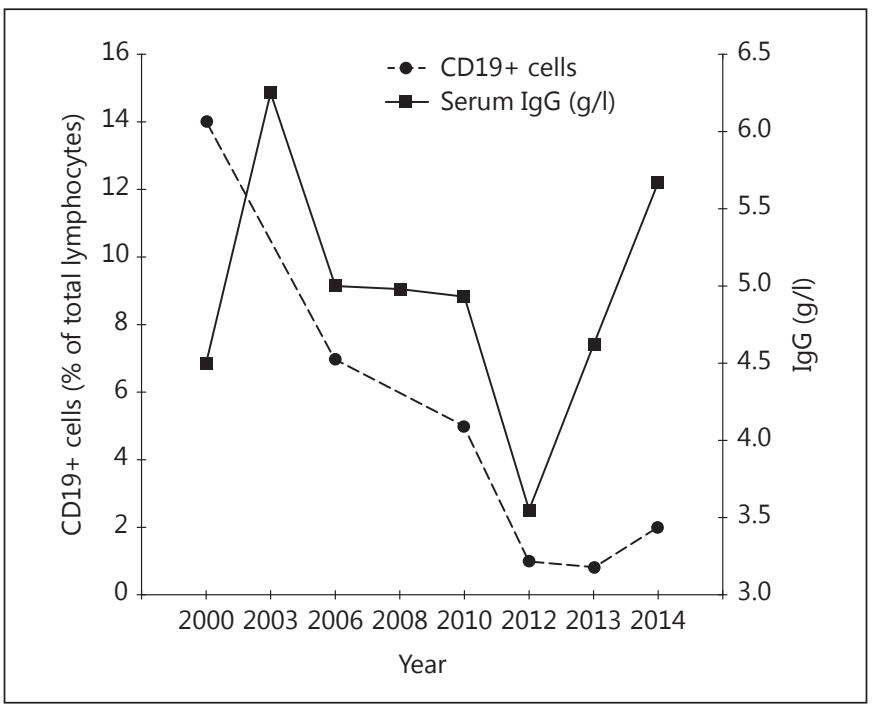

Fig. 3. Serum IgG levels and CD19+ cell numbers in patient 1. Progression of the number of CD19+ cells (\% of total lymphocytes) compared with serum IgG levels (g/l) since the diagnosis of CVID in 2000 and adjustment of immunoglobulin substitution to $10 \mathrm{~g}$ (164 mg/kg) per month in 2012.

during childhood, when he suffered from recurrent respiratory infections.

At 2 years of age, the patient had been admitted to hospital for severe ketoacidosis and hyperglycemia. Following a laboratory examination, he was diagnosed with T1D with negative T1D-specific antibodies (autoantibodies to GAD and IAA). An intensified insulin regimen was started, with a total daily dose of $57 \mathrm{IU}(1.04 \mathrm{IU} / \mathrm{kg} /$ day). Despite this regime, satisfactory compensation was not achieved, with glyc. HbA1c values repeatedly $>120 \mathrm{mmol} / \mathrm{mol}$, or $13.1 \%$. At 16 years of age, during a regular check-up, he showed signs of renal insufficiency (with an estimated GFR of $0.488 \mathrm{ml} / \mathrm{s}$ / $1.73 \mathrm{~m}^{2}$ ), and he was therefore admitted to the hospital for further examination. Laboratory testing (table 1 ) showed significant proteinuria $(1,036 \mathrm{mg} / 24 \mathrm{~h}$ ) and hematuria (13,333 erythrocytes/min). As part of the differential diagnosis, a kidney biopsy was performed; this biopsy showed changes that were typical of diabetic glomerulosclerosis/Kimmelstiel-Wilson nephropathy. The patient's stay in the hospital was further complicated by his development of peribronchitis, which required antibiotic therapy. An immunologic examination revealed severe hypogammaglobulinemia in the IgG $(0.76$ $\mathrm{g} / \mathrm{l}), \operatorname{IgA}(0.11 \mathrm{~g} / \mathrm{l})$ and $\operatorname{IgM}(0.04 \mathrm{~g} / \mathrm{l})$ classes and lower specific postvaccination antibodies. An autoantibody panel, cellular immunity and B lymphocyte subpopulations were also examined as part of the differential diagnosis. On the basis of the results (table 2), the patient was diagnosed with type II CVID via the Freiburg classification (fig. 1). Regular intravenous immunoglobulin substitution therapy totaling $10 \mathrm{~g}(169 \mathrm{mg} / \mathrm{kg})$ per month was started. During the patient's stay in the hospital, his metabolic control of T1D, including glycemic profiles, was satisfactory, and the immunoglobulin substitution was very well tolerated. Since his discharge from the hospital, we have not been able to acquire any new relevant data, as the patient did not attend any follow-up appointments due to noncompliance.

\section{Review of Literature and Data from the ESID Registry Database}

Here, we summarize our review of the literature and a targeted search of the ESID Registry Database. A total of 11 case reports involving a combination of T1D and CVID have been published since 1981 [13-22]. T1D was mentioned as a possible autoimmune complication of CVID in only 1 review article [23].

In summary, in most of the reports, the diagnosis of T1D preceded the CVID diagnosis by 7.5 years on average. The mean age at CVID manifestation was 18 years (range 5-39 years). Upper and lower respiratory tract infections were typically found in all patients, and infectious diarrhea and deep-skin infections were less common. In 5 cases (45\%), patients had to be treated for severe infections (including sepsis, pneumonia and meningoencephalitis). Autoimmune diseases other than T1D were found in 4 patients (37\%); inflammatory bowel disease in 2 and celiac disease and autoimmune cytopenia in 1 each. Mean age at cooccurrence of T1D manifestation was 11 years (range 11 months to 27 years). Ketoacidosis with hyperglycemia, polyuria with polydipsia, weight loss, fatigue and malaise were typical symptoms that led to the diagnosis of T1D. Similar to the observations in our 2 reported cases, familial occurrence of autoimmune diseases (including T1D) was found in 3 of the case reports published, and primary immunodeficiency (selective immunoglobulin A deficiency, sIgAD) was found in 1 family. T1D-specific antibodies were never detected in 3 previously described patients (27\%), and only 1 CVID patient with T1D tested positive for antibodies against islet cells. These antibodies were not assessed in the other patients who were diagnosed before 1982. In all of the published case reports, an intensified insulin regimen and an immunoglobulin replacement therapy were initiated. This complex therapeutic approach led to a reduced number of infections and improvements in metabolic control.

Similarly to CVID, T1D has been described in conjunction with other primary humoral immunodeficiencies, such as sIgAD or Bruton agammaglobulinemia (also known as XLA). The occurrence of T1D in SIgAD has been reported in many studies and varies from $0.5 \%(500 / 100,000)$ in Austria to $1.8 \%(1,800 / 100,000)$ in the UK [24]. Recent data from 2011 show an overall prevalence of T1D among sIgA patients of $1 \%(1,000 / 100,000)$ in a European Caucasian population and a roughly 5 -fold-higher prevalence in sIgAD patients compared with the incidence in the general population [25]. In contrast, the association of T1D and XLA has only been described in 1 case report, which described a 14-year-old male patient who developed T1D 11 years after the diagnosis of XLA; this patient had severely impaired antibody production [8].

Based on the findings in the literature, we initiated a targeted search of the ESID Registry database to obtain epidemiological data regarding the occurrence of T1D among CVID patients and to map the current situation. The ESID Registry is a Europe-wide database established in 2004 . To date, 19,355 patients with immunodeficiencies documented by 126 collaborating centers have been included. Overall, 1,671 of these patients were registered as having CVID, with 25 having co-occurrence of T1D and CVID. This number is equal to a $1.5 \%$ co-occurrence $(1,500 / 100,000)$. The highest number of patients was reported as being from in the UK $(n=5)$, the Netherlands $(n=4)$, Germany $(n=3)$ and the Czech Republic $(n=3)$. The remaining countries had fewer than 2 patients. 


\section{Discussion}

In this report, we describe $2 \mathrm{~T} 1 \mathrm{D}$ patients in whom CVID, unrecognized at the onset of the disease, was diagnosed later in its course and the associated autoantibodies were never detected. Our findings are supported by a review of the literature regarding T1D as an autoimmune manifestation of primary humoral immunodeficiency. The search revealed only 11 cases, in total, of co-occurrence of T1D and CVID since 1981 [13-22]. The association between T1D and CVID therefore seems to be uncommon. CVID is typically associated with other forms of autoimmunity, such as immune thrombocytopenic purpura and hemolytic anemia. Apart from above-mentioned case reports, T1D has been mentioned as a possible autoimmune complication of CVID in only 1 review article [23].

We assume that these case reports do not reflect the real incidence of T1D in CVID. In comparison with sIgAD, a humoral immunodeficiency also characterized by immune dysregulation with a higher coincidence of autoimmune diseases, the incidence of T1D is very wellcharacterized, with a 5-fold increase compared to the incidence in the general population [25]. CVID and sIgAD share some immunological background features, and sIgAD may progress to CVID in some patients; thus, a higher occurrence of T1D can be expected in CVID cohorts $[26,27]$. We therefore initiated a targeted search of the ESID registry database, which revealed that 25 of 1,671 listed CVID patients were reported as having T1D, meaning that the co-occurrence of T1D and CVID amounts to $1.5 \%(1,500 / 100,000)$.

Similarly to in our patients, most of the patients described had a substantially delayed diagnosis of CVID, with the average delay between T1D manifestation and CVID diagnosis being 7.5 years. Antibody deficiency was also reflected by missing T1D-associated antibodies. A total of $5 / 13$ patients $(38 \%)$, including ours, were diagnosed with T1D despite their lack of specific autoantibodies (i.e. anti-GAD, IAA and anti-IA2). In 5 patients diagnosed before 1982, the T1D-related associated autoantibodies were not assessed because autoantibodies to GAD were only discovered and found to be associated with type 1 diabetes in that year $[28,29]$.

The impaired antibody production with missing specific T1D-associated antibodies caused by the lack of an antigen-specific antibody response in our CVID patients with T1D was accompanied by significant changes in $B$ cell subsets, which were skewed in a fashion that is typical of CVID [30, 31]. In both patients, the B cell subset analysis (table 2) showed a significant reduction in switched- memory B cells and a slight decrease in plasmablasts. Furthermore, there was increased early maturation of these cells, such as in naïve and transitional B cells. Typical impairment of antibody secretion and maturation defects of B cells, however, does not rule out the possibility that the cells can interact with $\mathrm{T}$ lymphocytes. Indeed, impaired $\mathrm{T}$ - and B-cell interactions are considered to cause autoimmune complications in CVID.

Our findings confirm experimental data, suggesting that antibodies are not necessary for the development of T1D but indicating the importance of the presence of $B$ lymphocytes [32]. The importance of B lymphocytes in T1D is supported by a demonstration of the positive effect of B lymphocyte depletion in T1D in both mice and humans [33]. Therefore, although the basic function of B lymphocytes, i.e. the secretion of antibodies, only plays a secondary role in $\mathrm{T} 1 \mathrm{D}$ pathogenesis by serving as a diagnostic marker, other B lymphocyte functions may be vital for the development of the disease. Chief among these functions is antigen presentation by B lymphocytes and their interaction with CD4+ T lymphocytes, which leads to the proliferation and expansion of $\mathrm{T}$ lymphocytes and the T cell-mediated destruction of beta cells [34].

However, a case report showing T1D development on an XLA background with practically no peripheral B lymphocytes present contradicts the necessity of B lymphocytes for T1D development in humans. These findings open up the question about the complexity of immune reactions, including the role of innate immunity, in T1D development. Pathologies in innate immune responses, particularly in dendritic cells, have been described in both CVID and XLA patients [35]. We therefore assume that specific defects both in innate and adaptive immunity combine into a complex immune reaction and contribute to the autoimmune complications associated with CVID which include T1D.

The possibility of CVID and T1D co-occurrence also has clinical importance. The correct diagnosis of CVID and regular immunoglobulin substitution are crucial for immunodeficiency management, although the underlying immunodeficiency in cases of the co-occurrence of T1D and CVID may be overlooked. Less frequent occurrence and a milder clinical course of infections, improvements usually successfully achieved through immunoglobulin replacement therapy, may contribute to better T1D control. However, whether immunoglobulin replacement therapy can also directly influence T1D control is thus far unknown. The basic mechanism underlying the effect of immunoglobulin replacement therapy on CVID is the substitution of immunoglobulins, the pro- 
duction of which is impaired due to B lymphocyte defects or impaired B- and T-cell collaboration [36]. The immunomodulatory effect of immunoglobulins on autoimmune processes may also play a role. The main mechanism of immunomodulatory action is the production of anti-idiotype antibodies that neutralize autoreactive antibodies. Additionally, IVIG can inhibit antibody production and $\mathrm{B}$ cell differentiation, induce $\mathrm{B}$ cell apoptosis, reduce the production of proinflammatory cytokines, inhibit phagocytosis, increase the number and suppressive function of regulatory $\mathrm{T}$ cells or inhibit dendritic cells [37-41]. However, these processes have not yet been clearly documented, especially in connection with T1D as an autoimmune complication of CVID.

To answer these questions, additional investigation is needed. Thus far, we have mapped the current situation through a targeted search of the ESID registry, identifying 25 cases with a CVID and T1D combination. We plan to perform a more detailed study in collaboration with participating centers and to search for the specific immune background that leads to the development of autoimmune T1D in an immunodeficient environment.

\section{Conclusion}

Our cases demonstrate a combination of primary immunodeficiency, or CVID, with T1D and, according to our new data from the ESID registry, suggest that T1D is substantially more frequent among CVID patients than the estimated incidence in the general population. Our findings, supported by a literature review, also confirm that specific T1D-associated antibodies are not crucial for disease development. However, these results present questions regarding the necessity of B cells, their role in T1D pathogenesis as well as the role of innate immunity and the effect of immunoglobulin replacement therapy on T1D development. The possibility of the co-occurrence of T1D and CVID is also important from a clinical standpoint because the correct diagnosis and appropriate treatment of immunodeficiency not only improve the clinical state of the patient and decrease the frequency of concomitant infections, but may also contribute to better control of T1D. Basic immunologic investigations should complement a complex diagnostic process in T1D patients, particularly those with repeated infections.

\section{Acknowledgments}

This article was written with contributions from B. Gathmann on behalf of the ESID Registry Working Party and collaborating centers: B. Grimbacher (University College London), T.W. Kuijpers (Academisch Medisch Centrum, Amsterdam), E. Scharbatke (Universitätsklinikum Würzburg), B. Belohradsky (Ludwig/Maximilians Universität, München), A. Helbling (Inselspital, Bern), H. Longhurst (Bartshealth NHS), J. Litzman (University Hospital U Sv. Anny) and C. Bangs (Central Manchester University Hospitals). The study was supported by the Ministry of Health, Czech Republic - conceptual development of research organization, University Hospital Motol, Prague, Czech Republic (00064203).

\section{References}

1 Liese AD, D'Agostino RB Jr, Hamman RF, et al: The burden of diabetes mellitus among US youth: prevalence estimates from the SEARCH for Diabetes in Youth Study. Pediatrics 2006;118:1510-1518.

2 Patterson CC, et al: Incidence trends for childhood type 1 diabetes in Europe during 1989-2003 and predicted new cases 2005-20: a multicentre prospective registration study. Lancet 2009;373:2027-2033.

3 Herold $\mathrm{KC}$, et al: Type 1 diabetes: translating mechanistic observations into effective clinical outcomes. Nat Rev Immunol 2013;13: 243-256.

4 Cheng-Rui L, Bradley L: The role of Th17 cells in type 1 diabetes. J Immunol 2012;188:123130.

5 Lehuen A, et al: Immune cell crosstalk in type 1 diabetes. Nat Rev Immunol 2010;10:501513.
6 Notkins AL, Lernmark A: Autoimmune type 1 diabetes: resolved and unresolved issues. J Clin Invest 2001;108:1247-1252.

7 Atkinson MA, Eisenbarth GS: Type 1 diabetes: new perspectives on disease pathogenesis and treatment. Lancet 2001;358:221-229.

8 Martin S, et al: Development of type 1 diabetes despite severe hereditary B-lymphocyte deficiency. N Engl J Med 2001;345:10361040.

9 Jolles S: The variable in common variable immunodeficiency: a disease of complex phenotypes. J Allergy Clin Immunol Pract 2013;1: 545-556;quiz 557.

10 Bergbreiter A, Salzer U: Common variable immunodeficiency: a multifaceted and puzzling disorder. Expert Rev Clin Immunol 2009;5:167-180.

11 Deane $S$, et al: Common variable immunodeficiency: etiological and treatment issues. Int Arch Allergy Immunol 2009;150:311-324.
12 Cunningham-Rundles C: Autoimmune manifestations in common variable immunodeficiency. J Clin Immunol 2008;28(suppl 1):S42S45.

13 Catassi C, et al: Unresponsive enteropathy associated with circulating enterocyte autoantibodies in a boy with common variable hypogammaglobulinemia and type I diabetes. J Pediatr Gastroenterol Nutr 1988;7:608-613.

14 Conley ME, Park CL, Douglas SD: Childhood common variable immunodeficiency with autoimmune disease. J Pediatr 1986;108:915922.

15 Filipovic B, et al: Common variable immunodeficiency associated with inflammatory bowel disease and type I diabetes. Clin Med Case Rep 2009;2:67-71.

16 Iglesias P, Ferreira A, Diez JJ: Common variable immunodeficiency in an adult woman with IDDM. Diabetes Care 1998;21:1029. 
17 Johnston SL, Virgo PF, Unsworth DJ: Type 1 diabetes mellitus masking primary antibody deficiency. J Clin Pathol 2000;53:236-237.

18 Lopez Cruz MC, et al: Common variable immunodeficiency, insulin-dependent diabetes mellitus and celiac disease. Allergol Immunopathol (Madr) 2000;28:323-327.

19 Metin A, Tezcan I, Ozyurek H: IDDM in an adolescent patient with common variable immunodeficiency. Diabetes Care 1997;20:677678 .

20 Moffitt JE, et al: Type I diabetes in an adolescent with common variable immunodeficiency. J Allergy Clin Immunol 1989;84:191-196.

21 Yamaoka M, et al: A case of long-standing autoimmune type 1 diabetes with common variable immunodeficiency. Diabetol Int 2011;3:

22 Young RJ, et al: Development of insulin-dependent diabetes in adult-onset hypogammaglobulinaemia. Br Med J (Clin Res Ed) 1981; 282:1668.

23 Bussone G, Mouthon L: Autoimmune manifestations in primary immune deficiencies. Autoimmun Rev 2009;8:332-336.

24 Acerini CL, et al: Coeliac disease in children and adolescents with IDDM: clinical characteristics and response to gluten-free diet. Diabet Med 1998;15:38-44.

25 Wang N, et al: Selective IgA deficiency in autoimmune diseases. Mol Med 2011;17:13831396.
26 Aghamohammadi A, et al: Progression of selective IgA deficiency to common variable immunodeficiency. Int Arch Allergy Immunol 2008; 147:87-92.

27 Hammarstrom L, Vorechovsky I, Webster D: Selective IgA deficiency (SIgAD) and common variable immunodeficiency (CVID). Clin Exp Immunol 2000;120:225-231.

28 Baekkeskov S, Lernmark A: Rodent islet cell antigens recognized by antibodies in sera from diabetic patients. Acta Biol Med Ger 1982;41:1111-1115.

29 Baekkeskov S, et al: Autoantibodies in newly diagnosed diabetic children immunoprecipitate human pancreatic islet cell proteins. $\mathrm{Na}$ ture 1982;298:167-169.

$30 \mathrm{Al}$ Kindi M, et al: Utility of peripheral blood $B$ cell subsets analysis in common variable immunodeficiency. Clin Exp Immunol 2012; 167:275-281.

31 Vlkova M, et al: Characterization of lymphocyte subsets in patients with common variable immunodeficiency reveals subsets of naive human B cells marked by CD24 expression. J Immunol 2010;185:6431-6438.

32 Wong FS, et al: Investigation of the role of Bcells in type 1 diabetes in the NOD mouse. Diabetes 2004;53:2581-2587.

33 Marino E, et al: B cell-directed therapies in type 1 diabetes. Trends Immunol 2011;32: 287-294.

34 Cox SL, Silveira PA: Emerging roles for B lymphocytes in type 1 diabetes. Expert Rev Clin Immunol 2009;5:311-324.
35 Sochorova K, et al: Impaired Toll-like receptor 8-mediated IL-6 and TNF-alpha production in antigen-presenting cells from patients with X-linked agammaglobulinemia. Blood 2007;109:2553-2556.

36 Durandy A, et al: Immunoglobulin replacement therapy in primary antibody deficiency diseases - maximizing success. Int Arch Allergy Immunol 2005;136:217-229.

37 Durandy A, et al: Intravenous immunoglobulins - understanding properties and mechanisms. Clin Exp Immunol 2009;158(suppl 1):2-13.

38 Ephrem A, et al: Expansion of CD4+CD25+ regulatory $\mathrm{T}$ cells by intravenous immunoglobulin: a critical factor in controlling experimental autoimmune encephalomyelitis. Blood 2008; 111:715-722.

39 Kessel A, et al: Intravenous immunoglobulin therapy affects $\mathrm{T}$ regulatory cells by increasing their suppressive function. J Immunol 2007; 179:5571-5575

40 Matucci A, Maggi E, Vultaggio A: Mechanisms of action of Ig preparations: immunomodulatory and anti-inflammatory effects. Front Immunol 2014;5:690.

41 Paquin-Proulx D, et al: IVIg immune reconstitution treatment alleviates the state of persistent immune activation and suppressed CD4 T cell counts in CVID. PLoS One 2013 8:e75199. 